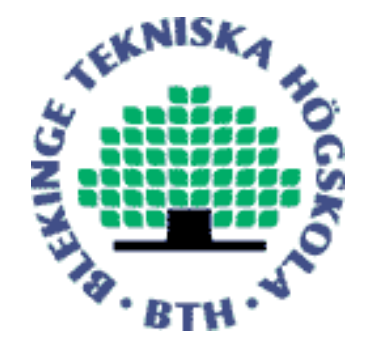

Copyright (C) 2012 IEEE.

Citation for the published paper:

Visual Quality Improvement of Digital Video by Stabilization Using Adaptive CMAC Filtering

Amir Zahoor, Wittaya Koodtalang,, Muhammad Shahid, Benny Lövström

6th International Conference on Signal Processing and Communication Systems (ICSPCS)

2012 Gold Coast, Australia

This material is posted here with permission of the IEEE. Such permission of the IEEE does not in any way imply IEEE endorsement of any of BTH's products or services Internal or personal use of this material is permitted. However, permission to reprint/republish this material for advertising or promotional purposes or for creating new collective works for resale or redistribution must be obtained from the IEEE by sending a blank email message to pubs-permissions@iee.org.

By choosing to view this document, you agree to all provisions of the copyright laws protecting it. 


\title{
Visual Quality Improvement of Digital Video by Stabilization Using Adaptive CMAC Filtering
}

\author{
Amir Zahoor, Wittaya Koodtalang, Muhammad Shahid and Benny Lövström \\ School of Engineering, Blekinge Institute of Technology \\ 371 79, Karlskrona, Sweden \\ Email: $\{$ amir.zahoor, muhammad.shahid $\} @$ ieee.org
}

\begin{abstract}
A digital Video Stabilization (DVS) system removes the unwanted shaking in the videos acquired by hand-held cameras and preserves the panning. In this paper, a digital video stabilization system is proposed based upon adaptive cerebellar model articulation controller (CMAC) filtering. A CMAC is a manifestation of the associative memory learning structure present in the cerebellum of human being. Adaptive CMAC filtering has favorable properties of small size, good generalization, rapid learning and dynamic response. Thus, it is more suitable for high-speed signal processing applications. The adaptive CMAC is used to adjust the coefficients of IIR filter employed in the proposed model. The training of CMAC is based upon fuzzy rule. The efficacy of the proposed adaptive CMAC filtering has been validated by evaluating it on a set of test video sequences.
\end{abstract}

\section{INTRODUCTION}

Light weight digital cameras and mobile phones are commonly used in daily life to capture videos. Videos acquired by these cameras can have undesired motion due to unstable camera holding and platform movement and desired panning. The challenge is how to compensate or remove the unwanted shaking without affecting moving objects to improve the perceptual quality of the image sequences [1]. Digital video stabilization (DVS) system is needed to remove shaking in the video acquired by hand cameras that are hand held or mounted on unstable platforms. It is also required to preserve panning of the camera and a system, which can perform all these tasks, has to compute on high speed.

A digital video stabilization system is divided into two major parts as shown in Fig. 1. First part is motion estimation that estimates the motion between two consecutive frames of the given video and generates global motion vector (GMV). Second part is motion compensation that compensates the shaky motion in video and produces a stabilized video.

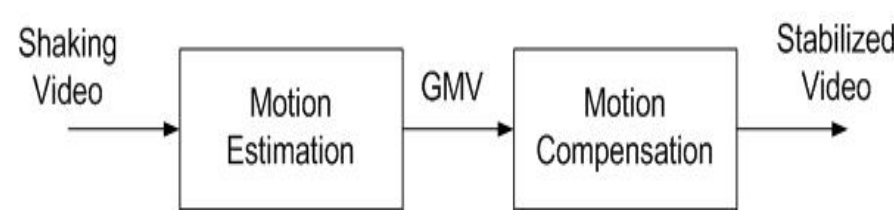

Fig. 1: Block diagram of digital video stabilization [2]

There exist some DVS systems to stabilize a shaky video. These systems have some challenges that are needed to be addressed, such as DVS system by using Kalman filter [3] may weaken stabilization because it treats some shaking phenomena as panning motion. DVS system by using Modified PI (MPI) controller [2] uses compensation motion vector (CMV) estimation to tune the parameter but when an integration gain $k_{i}$ is too large, then shaking stabilization will be destroyed. A DVS system by using Adaptive Fuzzy Filtering [4] requires more prior knowledge and has slow computational speed.

A CMAC is an adaptive system by which control functions for many degrees of freedom operating simultaneously can be computed by referring to table rather than by mathematical solution of simultaneous equations [5]. It can be considered as an associative memory learning structure based on the performance of the cerebellum of human being. The function of CMAC is similar to a lookup-table technique which represents complex and nonlinear systems [6]. CMAC was proposed for the identification and control of complex dynamic systems, due to its fast learning property and good generalization capability [7]. The structure of CMAC is shown in Fig. 2.

As CMAC filtering has useful properties of having small size, good generalization, rapid learning, and dynamic response. Therefore, this algorithm is suitable for high speed signal processing [8]. In this paper, a new model of DVS system is proposed that is based upon the CMAC filtering. Representative point matching method and background detection algorithm has been used to estimate the global motion vector (GMV). Adaptive CMAC filtering and IIR are used to estimate compensation motion vector (CMV). The proposed model has been applied to various videos with different irregular conditions. The experimental results indicate that proposed model performs well in all such conditions.

\section{The Proposed Model}

The proposed model of DVS system is illustrated in Fig. 3. We have a shaky video sequence as input of the proposed model. Local motion estimation determines the local motion vector (LMV) and ill-condition motion vector (IMV). Global 


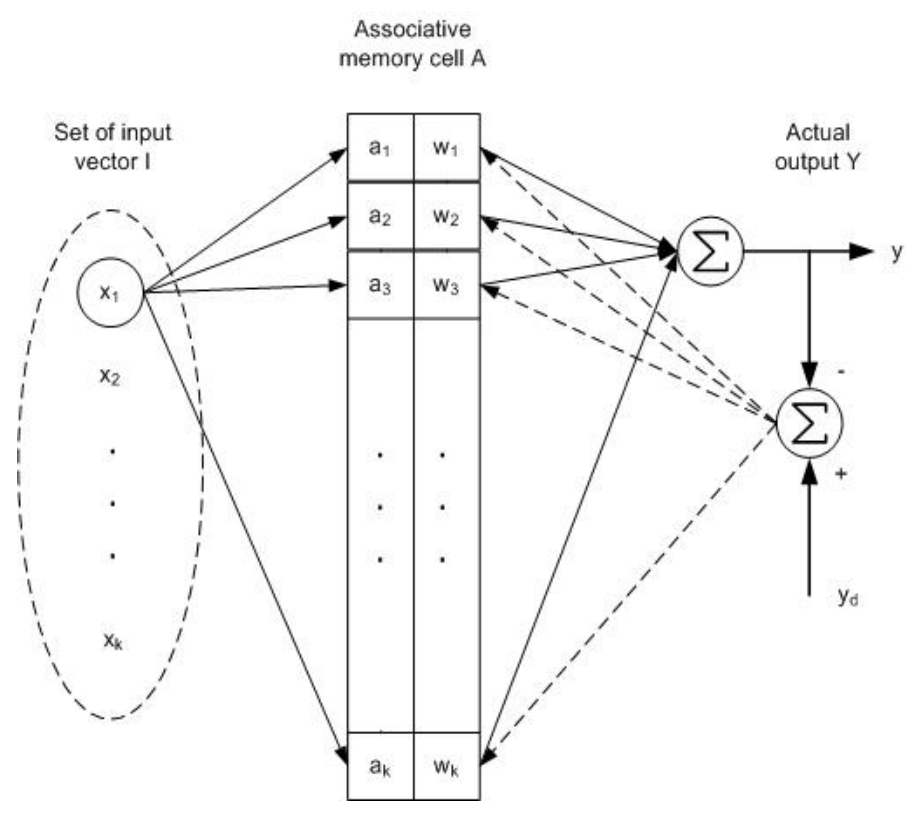

Fig. 2: CMAC architecture [9]

motion vector (GMV) estimates are done by using LMV and ILM. We applied adaptive CMAC on GMV to update the coefficient of the IIR filter. Compensation motion vector (CMV) is estimated by using IIR filtering. Unwanted motion estimation is done to generate the unwanted motion vector (UMV) which is used for motion correction. Finally, motion correction is used to find trajectories, which are used to compensate the sequences to get stabilized video. The details of each step are described in the sequel.

\section{A. Local Motion Estimation}

At the local motion estimation stage, the representative point matching (RPM) algorithm [1] is used. The RPM method divides the given video sequence into four regions. Each region is further divided into 30 sub-regions which consist of 5 rows and 6 columns [1]. The central point of each sub-region will be representing point $(\mathrm{X}, \mathrm{Y})$. The calculation of correlation of RPM is performed as given in following.

$$
R_{i}(p, q)=\sum_{r=1}^{N}\left|I\left(t-1, X_{r}, Y_{r}\right)-I\left(t, X_{r+p}, Y_{r+q}\right)\right|
$$

Where $N$ is the number of representative points in one region, $I$ is the intensity of each frame, $t$ is the frame number and i is the region. The local motion vector (LMV) for each region is calculate by

$$
L M V_{i}=(p, q) \quad \text { for } \quad R_{i M i n}=\min _{p, q}\left(R_{i}(p, q)\right)
$$

Where $R_{i}(p, q)$ is the correlation measure for shift $(p, q)$ between current frame and previous frame. $R_{i \text { Min }}$ is the minimum correlation value in region $i$.

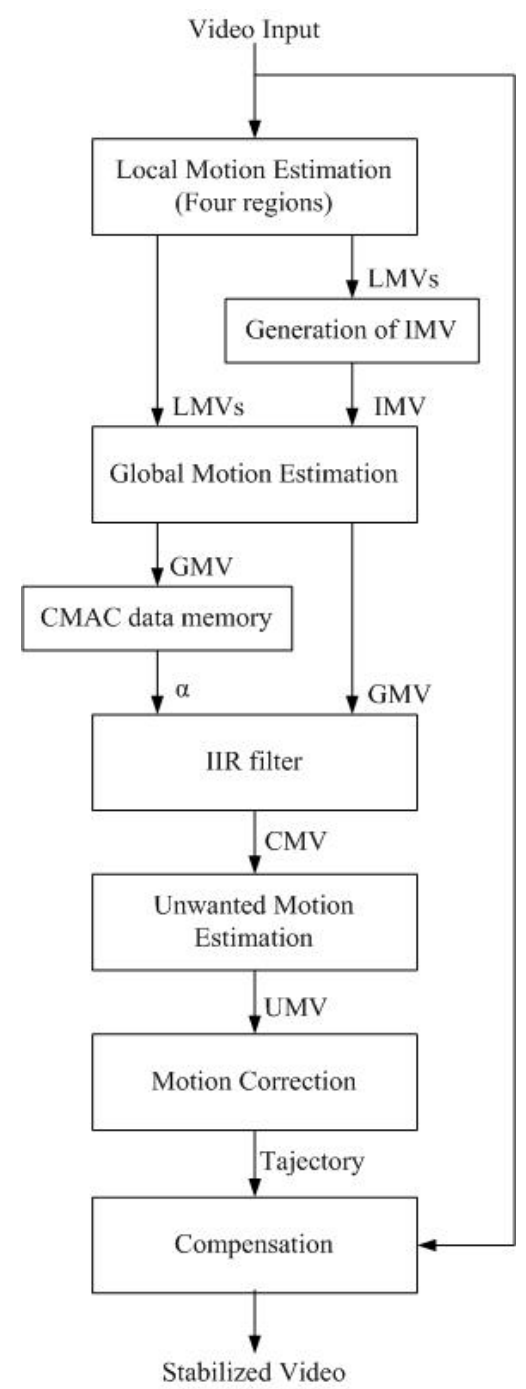

Fig. 3: Flowchart of the proposed DVS system

\section{B. Generation of Ill-Condition Motion Vector (IMV)}

The calculation process to generate ill-conditioned motion vector (IMV) has been proposed in [1] and is given in the following.

Firstly, calculate the number of $x_{i}$ and $y_{i}$ in four regions.

$$
\begin{aligned}
& \operatorname{Num}\left(x_{i}\right)=\operatorname{sum} \text { of }\left(x_{i} \text { is reliable }\right) \\
& \operatorname{Num}\left(y_{i}\right)=\operatorname{sum} \text { of }\left(y_{i} \text { is reliable }\right)
\end{aligned}
$$

When the confidence level of $x_{i}$ or $y_{i}$ is less than set threshold, then $x_{i}$ or $y_{i}$ is called reliable [1].

Secondly, the calculations to determine the IMV are given as:

Case 1. If $\operatorname{Num}\left(x_{i}(t)\right)=4$ then

$$
V_{i l l_{x}}(t)=\operatorname{Med}\left(V_{a_{x}}(t), V_{b_{x}}(t), V_{c_{x}}(t), V_{d_{x}}, G M V_{x}(t-1)\right.
$$

Case 2. If $N u m\left(x_{i}(t)\right)=3$ then

$$
V_{i l l_{x}}(t)=\operatorname{Med}\left(V_{a_{x}}(t), V_{b_{x}}(t), V_{c_{x}}(t)\right.
$$


Case 3. If $\operatorname{Num}\left(x_{i}(t)\right)=2$ then

$$
V_{i l l_{x}}(t)=\operatorname{Med}\left(V_{a_{x}}(t), V_{b_{x}}(t), G M V_{x}(t-1)\right.
$$

Case 4. If $\operatorname{Num}\left(x_{i}(t)\right)=1$ then

$$
V_{i l l_{x}}(t)=V_{a_{x}}(t)
$$

Case 5. If $\operatorname{Num}\left(x_{i}(t)\right)=0$ then

$$
V_{i l l_{x}}(t)=\gamma \times G M V_{\text {avgx }}(t-1)
$$

Where $\operatorname{Num}\left(x_{i}(t)\right)$ is the number of $\mathrm{x}$ component of reliable LMVs $V_{i l l_{x}}(t)$ is the $\mathrm{x}$ component of IMV, $V_{a_{x}}(t), V_{b_{x}}(t), V_{c_{x}}(t)$ and $V_{d_{x}}(t)$ represent $x$ component of reliable LMVs in four regions, respectively. $\operatorname{Med}()$ is a function of median operation, $t$ is the frame number and $\gamma$ is attenuation coefficient, $0<\gamma<1$. The $G M V_{\text {avg }}(t)$ can be calculate by

$$
G M V_{a v g x}=\varphi G M V_{a v g x}(t-1)+(1-\varphi) G M V_{x}(t) ;
$$

Then by applying similar operation on $y$ component, the resultant IMV is expressed as:

$$
V_{i l l}=\left[\begin{array}{l}
V_{i l l_{x}}(t) \\
V_{i l l_{y}}(t)
\end{array}\right]
$$

\section{Global Motion Estimation}

The five vectors (four LMV and one IMV) obtained from previous calculations are called pre-selected motion vectors (MV). The summation of absolute difference (SAD) has been proposed to select the high probabilities from pre-selected MV. The GMV estimation becomes difficult in the cases when a video contains large number of moving objects. The reason behind is that the estimation process has to readily switch between moving objects and the background [1]. A background-based evaluation function is shown in Fig. 4.

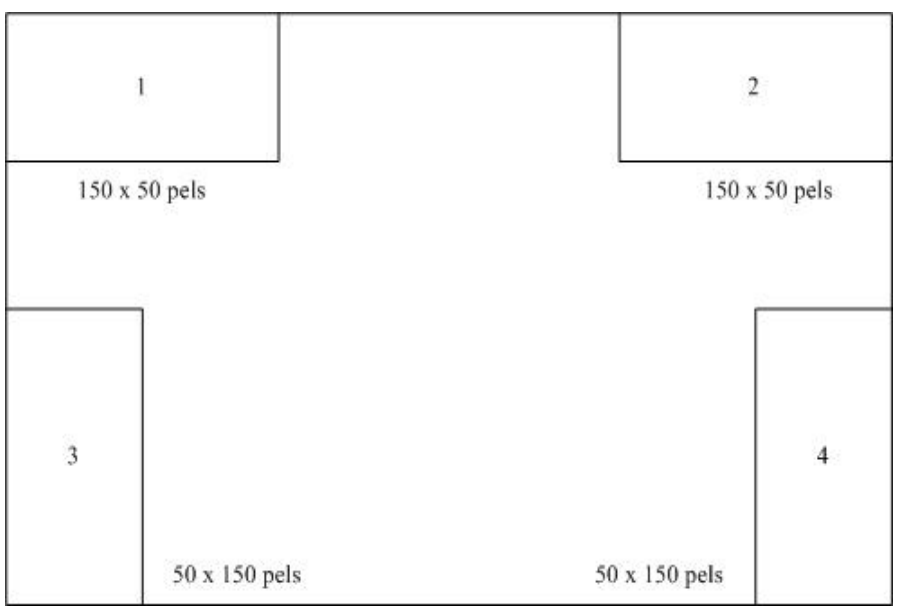

Fig. 4: Area of background detection and evaluation [1]

The summation of absolute difference (SAD) for GMV estimation in each background region is calculate by where $B_{i}$ is the $\mathrm{i}$-th background region, $I$ is the intensity of video frame, $X$ and $Y$ are the pixels coordinate, $X_{c}$ and $Y_{c}$ are the component in pre-selected $\mathrm{MV}$ and $t$ is the frame number. The smaller $S A D_{\left(B_{i}, c\right)}$ represent the higher probability of desire motion vector among these pre-selected MV. The higher $S A D_{\left(B_{i}, c\right)}$ indicates the higher score denote by $S_{(i, c)}$ and the total score for each pre-selected MV follow by

$$
S_{c}=\sum_{i=1}^{4} S_{i, c}
$$

Finally, GMV can be obtained by

$$
G M V=\text { pre-sel-MVini }- \text { th, for } R_{i M i n}=\arg _{c}\left(\min S_{c}\right)
$$

\section{Adaptive CMAC Filtering and Data Memory}

Adaptive CMAC algorithm is used to find the filter coefficient for the first-order IIR filter. The parameter is a smoothing factor. The larger filter coefficient $(\alpha)$ value leads to the higher smoothing factor. Therefore, a fixed valued of is not useful to good stabilized video.There are two input data for training in CMAC. First one denotes the noise in video frame which has range from 0 to 1.25 and the second one is denoted as acceleration which has range from 0 to 1 . Adaptive CMAC algorithm is used to train the network and keep the data into memory under fuzzy rule. The membership function generated by CMAC is shown in Fig. 5.

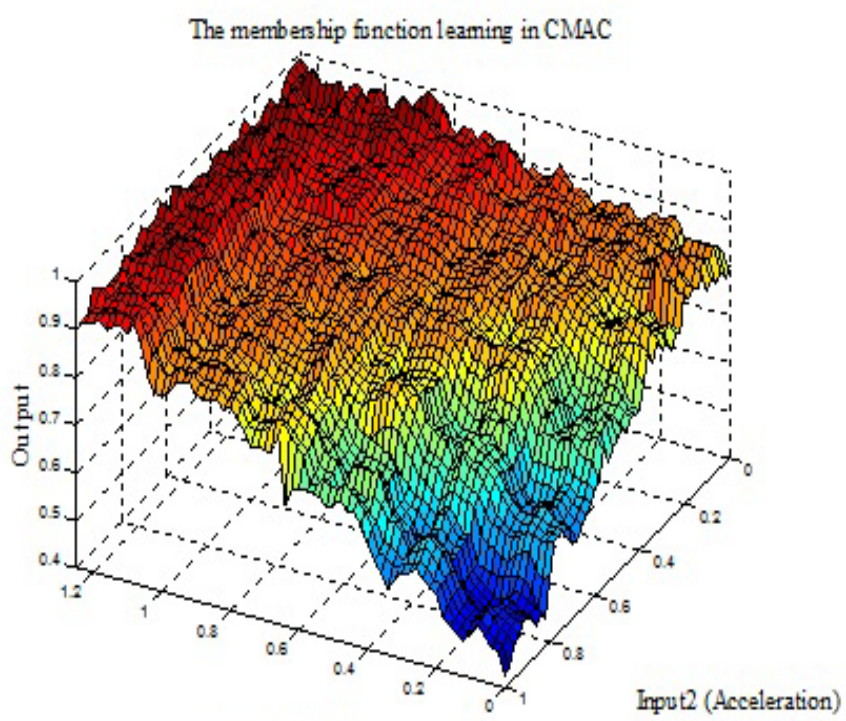

Inputl (Noise)

Fig. 5: The membership function learning in CMAC

The training will be stopped as error will be equal to or smaller than threshold level and we have set the threshold level for this research equal to $1 \times 10^{-} 11$. We are using fuzzy rule [4] to generate the output and use CMAC to learn about the output. It is also desired that memory size should be 
smaller. Our memory size is 2,000 memory locations to keep data small. The algorithm of CMAC learning is expressed as:

$$
m_{k+1}=m_{k}+\mu\left(x_{d}-x_{n}\right)^{2}
$$

where $m$ is the memory in CMAC, $\mu$ is the step size, $x_{d}$ is desired input and $x_{n}$ is the predicted output which can be calculated by determining the mean value in CMAC data memory for each input. The CMAC learning will stop as error becomes equal to or less than the threshold level. For video sequence number one, as given in the table 1, the learning error is shown in Fig. 6.

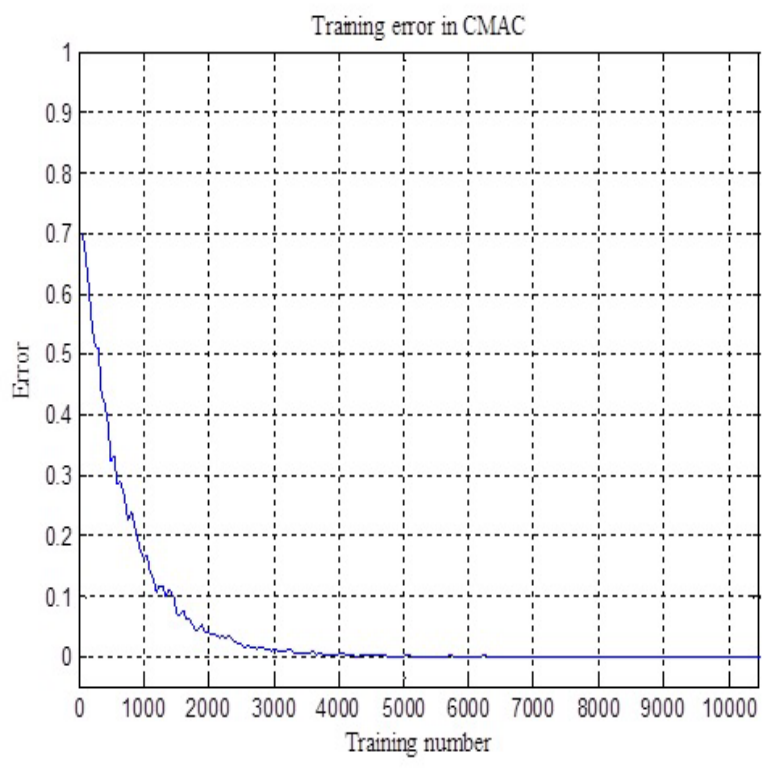

Fig. 6: Training error in CMAC

The CMAC data memory is created and it can be addressed directly when the system has known about noise and acceleration. Noise and acceleration can be calculate by

$$
\begin{aligned}
& \text { Noise }=\frac{1}{3} \sum_{i=t-2}^{t}|G M V(i)-G M V(i-1)| \\
& \text { Acceleration }=|G M V(t)-G M V(t-3)|
\end{aligned}
$$

Both noise and acceleration factors point to a certain memory position in CMAC and the $\alpha$ will be as outcome.

\section{E. Infinite Impulse Response (IIR) Filtering}

An estimated GMV may consist of two major components i.e. handshake and panning [4]. A good motion correction needs to remove shaking and preserve panning. Assuming that the handshake components are high frequency, the low-pass filter will be used for compensation motion estimation calculation. The proposed method to calculates the compensation motion vector (CMV) is expressed in the form of first-order autoregressive system as given by the following.

$$
C M V=\alpha C M V(t-1)+(1-\alpha) G M V(t)
$$

where $\alpha$ is the IIR filter coefficient which has calculated by CMAC and the index $t$ indicates the frame number. The reasons of using a first-order IIR filter are:

- It can be used in real time system,

- It requires small memory,

- It has low computational complexity,

- The compensation motion vectors produced by the filter are satisfactory to humans eye if suitable $\alpha$ is selected. For first three frames, $\alpha$ should have a fixed large value.

\section{F. Unwanted Motion Estimation and Motion Correction}

After computing the CMV by using the in CMAC data memory the unwanted motion vector (UMV) is obtained by

$$
U M V(t)=G M V(t)-C M V(t)
$$

To restore the current frame to its stabilized position, the motion trajectories can be obtained by

$$
\operatorname{Mtraj}(t)=\sum_{i=1}^{t} U M V(i)
$$

where Mtraj is the compensated motion trajectories of the video at frame $t$.

\section{G. Motion Compensation}

After calculation of motion trajectories, the compensation to stabilized video is last process for this DVS system. The motion compensation is illustrated in Fig. 7.

The offset should be fixed higher than the maximum of

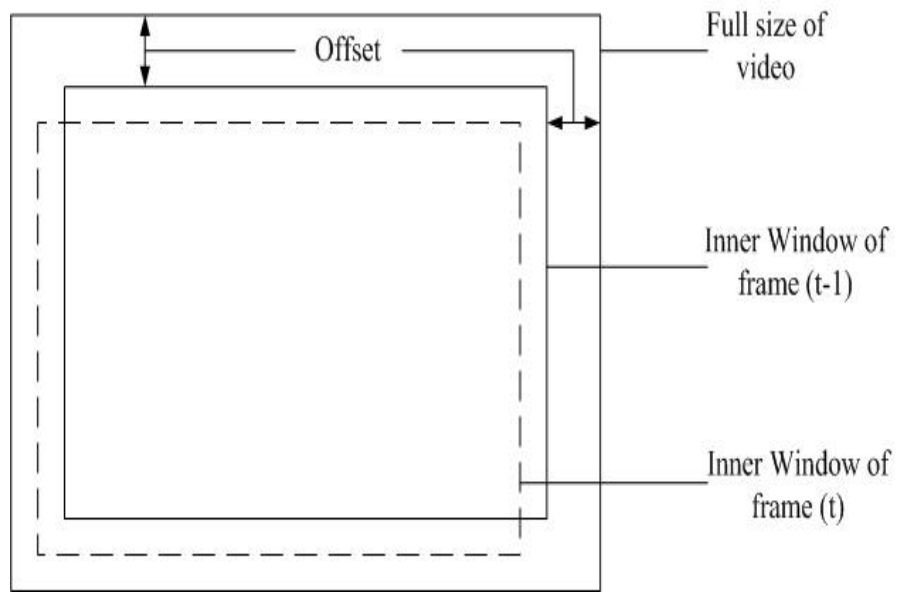

Fig. 7: The scheme of motion compensation [2]

absolute values in motion trajectories and the stabilized video can be obtained by

$$
\operatorname{Stabilized}(t)=\text { offset }-\operatorname{Mtraj}(t)
$$

$$
S t-\operatorname{Video}(t)=\text { OriginalVideo }(\text { Stabilized }(t))
$$

where of $f$ set is the shifting allowance which needs to be set before the calculation. The offset should be fixed higher than the maximum of absolute values in motion trajectories. $S t-V i d e o$ is the stabilized video. 


\section{EXPERIMENTAL RESULTS}

In this section, we evaluated the performance of proposed DVS system. MATLAB has been used as simulation platform. In our simulation, we tested four video sequences. First video was acquired from online source [10], second and third video were taken from MATLAB library [11], [12] and fourth video, named Wi-am, was captured by our own camera. Video names and their first frame image is shown in Table I.

TABLE I: Experimental video sequences and the image of their first frame

\begin{tabular}{|c|c|c|}
\hline $\begin{array}{c}\text { Video } \\
\text { No. }\end{array}$ & Video Name & First frame image \\
\hline 1 & Video-image-stabilization & \\
& & \\
2 & Quick-test-v-stabilization & \\
& & \\
\hline 3 & Shaky-car & \\
& & \\
\hline 4 & & \\
\hline
\end{tabular}

All these videos have different features and irregular conditions. These videos contain different number of frames with a resolution $480 \times 360$. Table II have more information about set of videos that have been used to stabilize.

TABLE II: Test video sequences information

\begin{tabular}{|c|c|c|l|}
\hline $\begin{array}{c}\text { Video } \\
\text { No. }\end{array}$ & Video Name & $\begin{array}{c}\text { Frame Rate } \\
\text { (fbs) }\end{array}$ & $\begin{array}{l}\text { Duration } \\
\text { (Sec.) }\end{array}$ \\
\hline 1 & Video-image-stabilization & 30 & 3.33 \\
\hline 2 & Quick-test-V-stabilization & 25 & 2.80 \\
\hline 3 & Shaky-car & 25 & 3.60 \\
\hline 4 & Wi-am & 25 & 4.80 \\
\hline
\end{tabular}

\section{A. Comparison of GMV and CMV Trajectories}

We also evaluate the adaptive CMAC filtering method to estimate CMV by four different GMV generated by set of shaky videos. Fig. 8 shows the comparison between shaky and compensated motion trajectories for every video. In each subfigure, blue line and red line show shaky and compensated trajectories respectively. GMV sets are estimated from video 1 to 4 and every video possessed different irregular conditions. here is comparison of trajectories of GMV and CMV in both
Fig. 8: Comparison of shaky and compensated motion trajectories between CMV (red line) and GMV (blue line) of following videos (a) Video-image-stabilization, (b) Quick-testv-stabilization, (c) Shaky-car and (d) Wi-am on each subfigure.

horizontal and vertical for all four videos. The results have shown that compensated horizontal trajectories, generated by adaptive CMAC and IIR filtering, are very close to horizontal shaky motion trajectories. Therefore, our proposed method can reduce the steady state lag and this gives more space to absorb the shakiness effect from video.

\section{B. Smoothness Index (SI)}

The sum of absolute difference $(S A D)$ of movements between two consecutive frames is used to determine the shaking effect of video. The value of image mass can be set from 0 to 1 according to degree of shakiness in the video measured by human visual perception. 
Smoothness index $(S I)$ is an important quantitative evaluation parameter for the performance of DVS system [2]. It is denoted as

$$
S I=\frac{1}{(N-1)} \sum_{t=2}^{N} m \times|G M V(t)-G M V(t-1)|
$$

where $N$ is the total number of frames, $t$ represents the frame number; $m$ is the image mass. Lower value of $S I$ means less shaking components in the image sequences Thus, Lower value of $S I$ is desired for any video. Hence, less shaking components i.e. small value $S I$ represents the better smoothness effect in any video. We took four shaky videos, possessed different irregular conditions, to apply the proposed CMAC filtering and determined the $S I$ for each video. In Table III, The results are shown that CMAC has improved $S I$ for all videos.

TABLE III: Experimental video sequences smoothness index

\begin{tabular}{|c|c|c|c|}
\hline $\begin{array}{c}\text { Video } \\
\text { No. }\end{array}$ & Coordinate & $\begin{array}{c}\text { CMAC Compensated } \\
(\text { SI })\end{array}$ & Original Shaky (SI) \\
\hline \multirow{2}{*}{1} & $\mathrm{x}$ & 0.8344 & 0.9241 \\
\cline { 2 - 4 } & $\mathrm{y}$ & 0.9358 & 1.0759 \\
\hline \multirow{2}{*}{2} & $\mathrm{x}$ & 3.2977 & 3.9130 \\
\cline { 2 - 4 } & $\mathrm{y}$ & 4.3851 & 5.9855 \\
\hline \multirow{2}{*}{3} & $\mathrm{x}$ & 2.3651 & 2.8090 \\
\cline { 2 - 4 } & $\mathrm{y}$ & 3.7509 & 4.6067 \\
\hline \multirow{2}{*}{4} & $\mathrm{x}$ & 0.8368 & 0.9496 \\
\cline { 2 - 4 } & $\mathrm{y}$ & 2.3263 & 3.0924 \\
\hline
\end{tabular}

\section{CONCLUSION}

In this paper, we proposed a new DVS system based upon adaptive cerebellar articulation controller (CMAC) which demonstrates a good stabilization performance. Global motion vector (GMV) is estimated by representative point matching (RPM) method and background detection algorithm. We applied the CMAC to determine the value of filter coefficient for the first order IIR filter which estimates the compensation motion vector (CMV). The simulation results show that the proposed DVS system achieves desired performance of video stabilization by preserving panning and removing shakiness from variety of videos. The proposed model has great significance for mobile video communication to improve the perceptual quality of digital video because of its low memory requirement. This method could be effective to produce stabilized video from shaky video sequences in video security monitoring, highway safety and live streaming applications. We have planed to test our method on a larger set of videos that possess a variety of resolutions and different levels of motion as a future work.

\section{REFERENCES}

[1] S.-C. Hsu, S.-F. Liang, and C.-T. Lin, "A robust digital image stabilization technique based on inverse triangle method and background detection," Consumer Electronics, IEEE Transactions on, vol. 51, no. 2, pp. $335-345$, may 2005 .
[2] C.-T. Lin, C.-T. Hong, and C.-T. Yang, "Real-time digital image stabilization system using modified proportional integrated controller," Circuits and Systems for Video Technology, IEEE Transactions on, vol. 19, no. 3, pp. $427-431$, march 2009.

[3] C. Wang, J.-H. Kim, K.-Y. Byun, J. Ni, and S.-J. Ko, "Robust digital image stabilization using the kalman filter," Consumer Electronics, IEEE Transactions on, vol. 55, no. 1, pp. $6-14$, february 2009.

[4] M. Tanakian, M. Rezaei, and F. Mohanna, "Digital video stabilization by adaptive fuzzy filtering," 19th European Signal Processing Conference (EUSIPCO 2011), vol. 19, no. 3, pp. 318-322, aug 2011.

[5] J. Albus, "A new approach to manipulator control: Cerebellar model articulation controller (cmac)," Transaction of the ASME, pp. 220-227.

[6] J. Juang and C. Lee, "Applications of cerebellar model articulation controllers to intelligent landing system," Journal of Universal Computer Science, vol. 15, pp. 2586-2607, 2009.

[7] C.-M. Lin and T.-Y. Chen, "Self-organizing cmac control for a class of mimo uncertain nonlinear systems," Neural Networks, IEEE Transactions on, vol. 20, no. 9, pp. $1377-1384$, sept 2009.

[8] C.-M. Lin, L.-Y. Chen, and D. Yeung, "Adaptive filter design using recurrent cerebellar model articulation controller," Neural Networks, IEEE Transactions on, vol. 21, no. 7, pp. 1149 -1157, july 2010.

[9] J. Juang and C. Lee, "Applications of cerebellar model articulation controllers to intelligent landing system," Journal of Universal Computer Science, vol. 15, no. 13, pp. 2586-2607, jul 2009.

[10] Oggencoder, "Video image stabilization," Available Online, sep 2008, http://en.wikipedia.org/wiki/File:Video image stabilization.ogv.

[11] MATLAB, "Video-image-stabilization," Available in Matlab toolboxvipblks-vipdemos.

[12] Matlab, "shaky-car.avi," Available in Matlab toolbox-vipblks-vipdemos. 